\title{
Déficit no autocuidado para banho: caracterização do diagnóstico de enfermagem em pacientes críticos
}

\author{
Deficit in self-care for bathing: characterization of the nursing diagnosis in critically ill \\ patients
}
Déficit en el autocuidado para el baño: caracterización del diagnóstico de enfermería en pacientes críticos

Luana Vieira Toledo ${ }^{1 *}$, Nádia Vaz Sampaio², Patrícia de Oliveira Salgado", Lídia Miranda Brinati ${ }^{3}$, Camila Santana Domingos ${ }^{1}$, Cristiane Chaves de Souza ${ }^{1}$, Flávia Falci Ercole ${ }^{4}$.

\section{RESUMO}

Objetivo: Estimar a prevalência das características definidoras e dos fatores relacionados ao diagnóstico de enfermagem "déficit no autocuidado para banho" entre os pacientes adultos críticos. Métodos: Trata-se de estudo transversal descritivo, conduzido com 51 pacientes internados em uma Unidade de Terapia Intensiva, entre fevereiro e abril de 2018. Os dados de caracterização sociodemográfica e clinica foram coletados no prontuário. Os dados referentes ao Diagnóstico de Enfermagem: Déficit no autocuidado para banho foram obtidos a partir da observação direta do paciente. Realizou-se a estatística descritiva. O estudo foi aprovado por Comitê de Ética em Pesquisa. Resultados: Houve um predomínio de pacientes idosos (33 - 64,7\%), do sexo masculino (28 - 54,9\%), considerados brancos (26 - 51,0\%), que evoluíram para a alta (43-84,3\%). A prevalência do "Déficit no autocuidado para banho" foi de 100,0\% entre os pacientes. A capacidade prejudicada para acessar a fonte de água, acessar o banheiro e pegar os artigos para banho foram as características definidoras apresentadas por todos os pacientes $(51-100,0 \%)$. Entre os fatores relacionados, o mais prevalente $(100,0 \%)$ foi a barreira ambiental. Conclusão: Conclui-se que os pacientes críticos possuem limitações para a manutenção da sua higiene corporal, sendo dependentes da equipe de enfermagem.

Palavras-chave: Enfermagem, Diagnóstico de enfermagem, Banho.

\begin{abstract}
Objective: To estimate the prevalence of defining characteristics and factors related to the nursing diagnosis "deficit in self-care for bathing" among critical adult patients. Methods: This is a descriptive cross-sectional study, conducted with 51 patients admitted to an Intensive Care Unit, between February and April 2018. Data on sociodemographic and clinical characterization were collected from the medical record. The data regarding the Nursing Diagnosis: Deficit in self-care for bathing were obtained from direct observation of the patient. Descriptive statistics were performed. The study was approved by the Research Ethics Committee. Results: There was a predominance of elderly patients (33 - 64.7\%), male (28 - 54.9\%), considered white (26-51.0\%), who progressed to discharge (43-84, 3\%). The prevalence of "Deficit in self-care for bathing" was $100.0 \%$ among patients. The impaired ability to access the water source, access the bathroom and take bath articles were the defining characteristics presented by all patients $(51-100.0 \%)$. Among the related factors, the most prevalent $(100.0 \%)$ was the environmental barrier. Conclusion: It is concluded that critical patients have limitations for maintaining their body hygiene, being dependent on the nursing team.
\end{abstract}

Keywords: Nursing, Nursing diagnosis, Baths.

\footnotetext{
1 Universidade Federal de Viçosa (UFV), Viçosa-MG. *E-mail: luana.toledo@ufv.br

2 Hospital das Cínicas (HC), Belo Horizonte -MG.

${ }^{3}$ Hospital São Sebastião (HSS), Viçosa-MG.

4 Universidade Federal de Minas Gerais (UFMG), Belo Horizonte-MG.
} 


\section{RESUMEN}

Objetivo: Estimar la prevalencia de características definitorias y factores relacionados con el diagnóstico de enfermería "déficit en el autocuidado para el baño" en pacientes adultos críticos. Métodos: Este es un estudio descriptivo de corte transversal, realizado con 51 pacientes ingresados en una Unidad de Cuidados Intensivos, entre febrero y abril de 2018. Los datos sobre caracterización sociodemográfica y clínica se obtuvieron de la historia clínica. Los datos sobre el diagnóstico de enfermería: déficit en el autocuidado para el baño se obtuvieron de la observación directa del paciente. Se realizó estadística descriptiva. El estudio fue aprobado por el Comité de Ética en Investigación. Resultados: Hubo un predominio de pacientes de edad avanzada (33 - 64.7\%), hombres (28 - 54.9\%), considerados blancos (26 - 51.0\%), que progresaron al alta (43 - 84, 3\%). La prevalencia de "Déficit en el autocuidado para el baño" fue del 100,0\% entre los pacientes. La capacidad deteriorada para acceder a la fuente de agua, acceder al baño y tomar artículos de baño fueron las características definitorias presentadas por todos los pacientes (51-100.0\%). Entre los factores relacionados, el más frecuente (100.0\%) fue la barrera ambiental. Conclusión: Se concluye que los pacientes críticos tienen limitaciones para mantener su higiene corporal, ya que dependen del equipo de enfermería.

Palabras clave: Enfermería, Diagnóstico de enfermería, Baños.

\section{INTRODUÇÃO}

O Processo de Enfermagem (PE) é um instrumento metodológico utilizado para orientar e organizar a assistência de enfermagem que possibilita a continuidade e a avaliação do cuidado oferecido. Este processo está organizado em cinco etapas inter-relacionadas e interdependentes denominadas de coleta de dados, diagnóstico de enfermagem, planejamento, implementação e avaliação. A realização dessas etapas subsidia a elaboração das intervenções do plano de cuidados direcionado ao atendimento das necessidades de cada paciente (BRASIL, 2009; TANNURE MC, PINHEIRO AM, 2011; FERREIRA AM, et al., 2016).

A coleta de dados além de constituir-se como a primeira etapa desse processo é fundamental para a execução das etapas seguintes, sobretudo a elaboração dos diagnósticos. A elaboração do Diagnóstico de Enfermagem (DE) está relacionada ao desenvolvimento do pensamento crítico do enfermeiro, ao passo que é construído a partir da interpretação e agrupamento dos dados obtidos durante coleta de dados (BRASIL, 2009; CHIANCA TCM, LIMA APS, SALGADO PO, 2012; BISPO MM et al., 2016).

Como forma de uniformizar a linguagem dos diagnósticos, a NANDA-Internacional (NANDA-I) tem desenvolvido um sistema conceitual para classificá-los em uma taxonomia (HERDMAN TH e KAMITSURU S, 2018).

Um estudo realizado com os pacientes internados em uma Unidade de Terapia Intensiva (UTI) de um município de Minas Gerais identificou que os diagnósticos da NANDA-I de risco de infecção, risco de constipação e déficit do autocuidado para banho estiveram presente em $100,0 \%$ dos pacientes (CHIANCA TCM, LIMA APS, SALGADO PO, 2012). Destaca-se que dois desses diagnósticos se relacionam a vulnerabilidade dos pacientes, sendo classificados como diagnósticos de risco. Em contrapartida, o terceiro diagnóstico direciona-se a um problema real relacionado à perda da autonomia para o autocuidado dos pacientes internados na UTI, o qual também foi evidenciado em outro estudo, realizado em uma UTI do estado de São Paulo (SALOMÉ GM, 2011).

O "Déficit no autocuidado para banho" é definido pela NANDA-I como a incapacidade de completar as atividades de limpeza do corpo de forma independente. A capacidade prejudicada de acessar a fonte de água, de acessar o banheiro, de lavar o corpo, de pegar os artigos para o banho, de regular a água do banho e incapacidade de secar o corpo são consideradas características definidoras desse diagnóstico. Em relação aos seus fatores relacionados estão incluídos a ansiedade, barreira ambiental, dor, fraqueza e motivação diminuída (HERDMAN TH, KAMITSURU S, 2018).

A aplicação efetiva do processo de enfermagem está relacionada à melhoria da qualidade da assistência oferecida, além do auxílio na construção de um corpo de conhecimentos, baseado em evidências de cuidado, na fundamentação do ensino, no gerenciamento de custos e na alocação de recursos para a qualificação dos serviços de enfermagem (HORTA FG, et al., 2014). Nesse contexto, apesar da existência de estudos que 
identificaram a elevada frequência do diagnóstico de déficit no autocuidado para banho em pacientes internados em UTI, faz-se necessário explorar os fatores relacionados e as características definidoras que confirmam a presença desse diagnóstico para o direcionamento das ações da equipe de enfermagem (SALOMÉ GM, 2011; CHIANCA TCM, LIMA APS, SALGADO PO, 2012).

Espera-se que a partir da identificação desses dados seja possível planejar uma assistência de enfermagem direcionada ao reestabelecimento da condição de saúde do paciente, com vistas ao alcance da sua capacidade de se autocuidar, sobretudo em relação à higiene corporal. Diante dessas considerações questiona-se: Quais os fatores relacionados e as características definidoras do diagnóstico de enfermagem "déficit no autocuidado para banho" entre os pacientes críticos mais prevalentes? Este estudo teve como objetivo estimar a prevalência das características definidoras e dos fatores relacionados ao diagnóstico de enfermagem "déficit no autocuidado para banho" entre os pacientes adultos críticos.

\section{MÉTODOS}

Trata-se de um estudo do tipo observacional, transversal, desenvolvido em uma UTI de Adultos de um hospital filantrópico de médio porte, localizado em um município da Zona da Mata Mineira, MG. A referida UTI é composta por seis leitos e direciona-se ao atendimento nas diversas especialidades clínicas e cirúrgicas.

A população de estudo foi composta por todos os pacientes adultos que estiveram internados na UTI entre os meses de fevereiro e abril de 2018 , totalizando 87 pacientes. Do total de pacientes internados nesse período, foram incluídos na amostra aqueles que foram submetidos à intervenção de enfermagem para a manutenção da sua higiene corporal, totalizando 51 pacientes. Os 36 pacientes não incluídos no estudo foram transferidos para outro hospital ou vieram a óbito antes de receberem a intervenção de banho pela equipe de enfermagem.

Para a coleta de dados foi utilizado um questionário no formato de checklist composto pelos dados de identificação (idade, data de nascimento, sexo, procedência, etnia, data de internação, data do desfecho), características da condição clínica, diagnóstico médico da internação (de acordo com a Classificação Internacional de Doenças (CID) e Problemas Relacionados à Saúde [CID-10]), comorbidades associadas, deformidades, sequelas psicológicas/físicas), características definidoras e fatores relacionados ao DE "Déficit no autocuidado para banho", propostos pela NANDA-I.

Os pesquisadores coletaram as informações do questionário a partir da observação direta do paciente e da consulta aos dados descritos no prontuário. Os dados foram duplamente digitados no programa Microsoft Excel e analisados utilizando-se o programa Statistical Package for Social Science for Windows (SPSS) versão 20.0. Realizou-se a estatística descritiva com distribuição de frequências simples e percentuais.

Este estudo foi aprovado pelo Comitê de Ética em Pesquisa da Universidade Federal de Viçosa (Parecer 2.259.675). Os pacientes inseridos no estudo foram orientados sobre os objetivos da pesquisa e convidados para participar da sua realização mediante a assinatura do Termo de Consentimento Livre e Esclarecido (TCLE). Nos casos em que os pacientes não foram capazes de assinar o TCLE, foi solicitada a assinatura do seu responsável. Foi resguardado o sigilo e anonimato dos pacientes e dos profissionais envolvidos

\section{RESULTADOS}

Dentre os 51 pacientes internados houve um predomínio de pacientes com idade $\geq 60$ anos (33-64,7\%), do sexo masculino (28 - 54,9\%), considerados etnicamente brancos (26 - 51,0\%). O tempo de permanência dos pacientes na UTI variou de dois a 28 dias, com média de 6,6 dias.

A maioria dos pacientes (26 - 51,0\%) foi encaminhada para a UTI pelo serviço de emergência. Entre as principais causas de internação destacam-se as doenças do aparelho circulatório (18 - 35,3\%), respiratório (9 - 17,6\%) e doenças infecciosas e parasitárias (06 - 11,8\%).

Em relação à história patológica pregressa, observou-se a presença de comorbidades em 39 pacientes (76,5\%). A alta da UTI foi o desfecho clínico mais prevalente (38 - 74,5\%), conforme descrito na Tabela 1. 
Tabela 1 - Análise descritiva dos dados sóciodemográficos e clínicos dos pacientes internados na Unidade de Terapia Intensiva. $n=51$. Viçosa - MG, 2018.

\begin{tabular}{|c|c|c|}
\hline Variáveis & $\mathbf{N}$ & Frequência (\%) \\
\hline \multicolumn{3}{|l|}{ Idade } \\
\hline 18 a 59 anos & 18 & 35.3 \\
\hline$\geq 60$ anos & 33 & 64,7 \\
\hline \multicolumn{3}{|l|}{ Sexo } \\
\hline Masculino & 28 & 54,9 \\
\hline Feminino & 23 & 45,1 \\
\hline \multicolumn{3}{|l|}{ Etnia } \\
\hline Branca & 26 & 51,0 \\
\hline Preta & 06 & 11,8 \\
\hline Parda & 19 & 37,3 \\
\hline \multicolumn{3}{|l|}{ Diagnóstico Médico na Internação (CID - 10) } \\
\hline 1. Algumas doenças infecciosas e parasitárias & 06 & 11,8 \\
\hline 2.Neoplasias (tumores) & 01 & 2,0 \\
\hline 4. Doenças endócrinas nutricionais e metabólicas & 04 & 7,8 \\
\hline 9. Doenças do aparelho circulatório & 18 & 35,3 \\
\hline 10. Doenças do aparelho respiratório & 09 & 17,6 \\
\hline 11. Doenças do aparelho digestivo & 05 & 9,8 \\
\hline 14. Doenças do aparelho geniturinário & 01 & 2,0 \\
\hline $\begin{array}{l}\text { 18. Sintomas, sinais e achados anormais de exames clínicos e de } \\
\text { laboratório, não classificados em outra parte }\end{array}$ & 05 & 9,8 \\
\hline $\begin{array}{l}\text { 19. Lesões, envenenamentos e algumas outras consequências de } \\
\text { causas externas }\end{array}$ & 02 & 3,9 \\
\hline \multicolumn{3}{|l|}{ Comorbidades associada } \\
\hline Não & 12 & 23,5 \\
\hline Sim & 39 & 76,5 \\
\hline \multicolumn{3}{|l|}{ Deformidades } \\
\hline Não & 47 & 92,2 \\
\hline Sim & 04 & 7,8 \\
\hline \multicolumn{3}{|l|}{ Sequelas Psicológicas/Físicas } \\
\hline Não & 50 & 98,0 \\
\hline Sim & 01 & 2,0 \\
\hline \multicolumn{3}{|l|}{ Desfecho Clínico } \\
\hline Alta & 43 & 84,3 \\
\hline Óbito & 08 & 15,7 \\
\hline
\end{tabular}

Fonte: Toledo LV, et al., 2020.

O Diagnóstico de Enfermagem "Déficit no autocuidado para banho" esteve presente em todos os 51 pacientes, assumindo uma prevalência de 100,0\%. Entre as características definidoras do referido diagnóstico, percebe-se que a capacidade prejudicada para acessar a fonte de água, acessar o banheiro e pegar os artigos para banho estiveram presentes em todos os pacientes $(51-100,0 \%)$. De forma semelhante, a barreira ambiental foi o fator relacionado mais prevalente (51-100,0\%). A Tabela 2 apresenta a descrição completa da prevalência das características definidoras e fatores relacionados do respectivo diagnóstico de enfermagem. 
Tabela 2 - Caracterização do Diagnóstico de Déficit no Autocuidado para banho entre os pacientes internados na Unidade de Terapia Intensiva. n=51. Viçosa - MG, 2018.

\begin{tabular}{lll}
\hline $\begin{array}{l}\text { Variáveis relacionadas ao Diagnóstico de Enfermagem: } \\
\text { "Déficit no autocuidado para banho" }\end{array}$ & $\mathbf{n}$ \\
\hline Características Definidoras do Diagnóstico & & \\
\hline Capacidade prejudicada de acessar fonte de água & 51 & 100 \\
Capacidade prejudicada de acessar o banheiro & 51 & 100 \\
Capacidade prejudicada de lavar o corpo & 48 & 94,1 \\
Capacidade prejudicada de pegar os artigos para banho & 51 & 100 \\
Capacidade prejudicada de regular a água do banho & 48 & 94,1 \\
Incapacidade de secar o corpo & 47 & 92,2 \\
\hline Fatores Relacionados ao Diagnóstico & & \\
\hline Ansiedade & 06 & 11,8 \\
Barreira ambiental & 51 & 100 \\
Dor & 09 & 17,6 \\
Fraqueza & 13 & 25,5 \\
Motivação diminuída & 05 & 9,8 \\
\hline
\end{tabular}

Fonte: Toledo LV, et al., 2020.

\section{DISCUSSÃO}

Nesse estudo houve um predomínio de internações de pacientes idosos, do sexo masculino, pertencentes à raça branca. A principal causa de internação foi por doenças do aparelho circulatório. Tais achados assemelham-se aos encontrado em pesquisas realizadas em diferentes UTIs brasileiras (CABRAL VH, et al., 2017; MELO ACL, MENEGUETI MG, LAUS AM, 2014; PERÃO OF, et al., 2016; QUEIRÓZ F, REGO D, NOBRE G, 2013).

Naturalmente o processo de envelhecimento é marcado pelo aparecimento de condições de desestabilização orgânica dos indivíduos que levam à necessidade de leitos de UTI, para atender às demandas desses doentes (MELO ACL, MENEGUETI MG, LAUS AM, 2014). Ademais, o elevado número de internação de idosos vem sendo associado à transição demográfica vivenciada, na qual há um progressivo envelhecimento da população (RAMOS GS, et al., 2013). De forma similar, as principais causas de internação identificadas nessa população refletem a elevada prevalência das doenças não transmissíveis, destacandose as doenças cardiovasculares (DCV).

As DCV vêm sendo consideradas como um problema de saúde pública devido ao impacto da sua morbimortalidade tanto para o indivíduo quanto para o sistema de saúde (GUIMARÃES RM, et al., 2015; SIQUEIRA ASE, SIQUEIRA-FILHO AG, LAND MGP, 2017).

Além das condições prévias à admissão na UTI, verificou-se os dados relacionados ao tempo de permanência e desfecho da internação. Nesse estudo, os pacientes permaneceram internados na UTI em média 6,6 dias, valor este menor do que o encontrado em uma pesquisa que avaliou o perfil de internações em três UTIs da cidade de Anápolis - GO, onde o tempo de permanência calculado em dias variou de um a 207 e a média foi de 7,6 dias (CASTRO RR et al., 2016). Por outro lado, uma pesquisa documental realizada com 695 prontuários de pacientes internados em uma UTI de Florianópolis - SC identificou uma média de permanência de seis dias (RODRIGUEZ AH, et al., 2016).

A literatura científica aponta que diferentes fatores podem interferir no tempo de permanência dos pacientes na UTI, destacando-se dentre eles a relação diretamente proporcional entre pacientes idosos, com comorbidades e maior média de permanência. (DIETRICH C, et al., 2017). Cabe ressaltar que a permanência prolongada na UTI, por si só, pode afetar negativamente o estado de saúde dos pacientes interferindo, assim, no seu desfecho clínico (BEZERRA GKA, 2012). 
Nessa investigação, a maior parte dos pacientes (43 - 84,3\%) evoluiu positivamente durante a internação e recebeu alta da UTI, corroborando com os achados de outras pesquisas na qual a alta foi o desfecho clínico mais prevalente entre os pacientes internados em UTIs (CASTRO RR, et al., 2016, RODRIGUEZ AH, et al., 2016). Nesse sentido, destaca-se a importância do papel dos profissionais intensivistas no reestabelecimento da condição de saúde dos pacientes críticos e maior autonomia. Uma equipe qualificada pode ser capaz de conduzir os pacientes a desfechos clínicos favoráveis apesar do grau de severidade inicial dos pacientes.

Paralelamente à avaliação da gravidade dos pacientes deve ser realizada a análise do grau de dependência em relação à satisfação das necessidades humanas básicas (NHB), como é o caso da higiene corporal.

A necessidade de cuidado corporal é definida como a necessidade do indivíduo para realizar atividades com o objetivo de preservar o seu asseio corporal de forma deliberada, responsável e eficaz (BENEDET AS, BUB MBC, 2001). Esta necessidade está diretamente relacionada à autonomia do indivíduo para o desempenho das atividades básicas de autocuidado, incluindo-se a capacidade para alimentar-se, para vestirse e para o cuidado da sua higiene pessoal (PORTO MLL, NÓBREGA MML, SANTOS SSC, 2005). O enfermeiro, ao oferecer assistência ao paciente com a necessidade de cuidado corporal comprometida deve identificar a existência de um problema de enfermagem e elaborar um plano de cuidados que contemple uma intervenção.

A presença do Diagnóstico de Enfermagem "Déficit no autocuidado para banho" pode ser considerado um indicador do quanto esses pacientes conseguem ou não, serem independentes na satisfação de sua necessidade de higiene. Nesse estudo a prevalência desse diagnóstico foi de 100,0\%, caracterizando a incapacidade do paciente em realizar o próprio autocuidado, tornando-os dependentes da equipe de enfermagem para a sua execução.

Estudos nacionais e internacionais reforçam a elevada frequência desse diagnóstico entre os pacientes críticos, o que confirma a importância do cuidado corporal como uma intervenção de enfermagem rotineira nos serviços de alta complexidade como as UTIs (ROSIN J, et al., 2016; OKUMA SM, et al., 2017; MARTINS CP, et al., 2018; PEDRÃO TGG, et al., 2018).

Ações direcionadas à promoção da higiene corporal são consideradas atividades rotineiras para os indivíduos saudáveis, no entanto para os pacientes internados em UTIs, a gravidade do seu quadro clínico aliado à perda da sua autonomia faz dessa atividade uma necessidade de cuidado profissionalizado que merece atenção da equipe de enfermagem (COSTA GS, et al., 2018). Para que essa intervenção da equipe de enfermagem seja efetiva e possa de fato modificar o quadro de déficit no autocuidado faz-se necessário 0 reconhecimento das características definidoras desse diagnóstico.

Reconhecer as inferências observáveis, que se agrupam como manifestações desse diagnóstico torna-se importante para avaliar a efetividade das ações da equipe de enfermagem (HERDMAN TG, KAMITSURU S, 2018). Entre as características que definem a presença do diagnóstico, percebe-se que a capacidade prejudicada de acessar a fonte de água, o banheiro e de pegar os artigos para banho esteve presente em todos os pacientes críticos avaliados (51 - 100,0\%). Essa evidência reforça a condição de dependência dos pacientes que são admitidos na UTI, os quais, na maioria das vezes, estão permeados de dispositivos invasivos que dificultam a sua locomoção até mesmo no leito e aumentam o risco de complicações (MAILLET TH, et al., 2014; SOUSA MAS, et al., 2017).

Para o planejamento da assistência de enfermagem, além do reconhecimento das manifestações do diagnóstico, o enfermeiro deve ter ciência da etiologia do problema, ou seja, quais são os fatores que estão relacionados a ocorrência desse déficit no autocuidado (HERDMAN TH, KAMITSURU S, 2018). Nesse estudo, a barreira ambiental foi o fator relacionado prevalente em todos os pacientes (51 - 100,0\%). Tal variável remete a questões próprias do ambiente da UTI, onde são encontradas facilidades e dificuldades para a realização das intervenções de enfermagem.

A UTI é considerada uma área específica, diferenciada dos outros ambientes pela sua densidade tecnológica. O seu ambiente fechado, organizado e controlado é também visto como complexo e desconhecido para os pacientes e familiares (BACKES MTS, ERDMANN AL, BUSCHER A, 2015). Os 
pacientes que ali se encontram, vivenciam um momento onde tudo passa a ser decidido pelo outro, como a hora da alimentação, de tomar banho, de receber visitas e de ser manuseado. (EULÁLIO MC, et al., 2016).

Além de interferir na capacidade de ações de autocuidado para a promoção da higiene corporal, a barreira ambiental pode ser considerada como um dos fatores relacionados à não realização da mobilização precoce entre os pacientes gravemente enfermos. Uma pesquisa realizada em dois hospitais de ensino do Rio Grande do Sul evidenciou desafios relacionados ao ambiente da terapia intensiva para a realização da mobilização precoce (FONTELA PC, FORGIARINI JUNIOR LA, FRIEDMAN, 2018).

Os demais fatores relacionados ao DE como: ansiedade, dor, fraqueza e motivação diminuída podem ter relação não apenas com a condição clínica do paciente, mas com os sentimentos vivenciados durante a experiência da internação em um serviço de alta complexidade. Nesse sentido, os profissionais de enfermagem, além de intervirem sobre os aspectos biológicos, não devem ignorar que os pacientes críticos ao serem submetidos aos procedimentos de higiene corporal podem apresentar alterações psíquicas em decorrência da vulnerabilidade e medo do desconhecido. O enfermeiro deve garantir que as informações sejam claras, objetivas e precisas para que estas alterações possam ser minimizadas (LOPES JL, et al., 2015).

A equipe de enfermagem, tomando por base a avaliação da condição clínica, severidade e o grau de dependência dos pacientes, deve ser capaz de promover uma assistência efetiva na manutenção da higiene corporal, buscando o reestabelecimento do equilíbrio das necessidades humanas básicas.

Nos pacientes considerados clinicamente estáveis e com certa autonomia essa assistência pode ser realizada sob a forma de banho de aspersão, que consiste no banho que o paciente senta ou fica em pé sob uma água corrente contínua (POTTER PA, 2017). Por outro lado, os pacientes com maior gravidade e instabilidade devem receber o banho sob a forma de banho no leito, tradicionalmente realizado a partir de compressas umedecidas, utilizando-se bacias com água e sabão (COSTA GS, et al. 2018). Para esses pacientes a satisfação de sua necessidade humana básica compete aos profissionais de enfermagem, devido ao grande comprometimento da capacidade de autocuidado (DIAS JAA, et al., 2016).

O banho no leito pode ser dividido em banho completo e banho parcial. A escolha pelo tipo de banho a ser realizado dependerá de uma avaliação criteriosa do estado clínico do paciente. O banho completo no leito compreende a higiene de todo o corpo do paciente pela equipe de enfermagem.

Esse tipo de banho destina-se à manutenção da higiene corporal de pacientes com maior grau de dependência, que não conseguem sozinhos realizar tal atividade. Por sua vez, o banho parcial, realizado com pacientes restristos ao leito, mas com certo grau de autonomia, inclui a ajuda do profissional de enfermagem apenas para a realização da higiene de partes do corpo, as quais não podem ser alcançadas pelos próprios pacientes (POTTER PA, 2017).

O banho no leito traz diversos benefícios ao paciente como: higienização da pele com redução de odores desagradáveis, estimulação sanguínea, movimentação, recuperação da autoimagem e alívio de desconforto. Consiste também no momento ideal para uma avaliação completa do paciente através da observação e do toque (BACKES DS, et al., 2017). No entanto, o banho não é uma atividade isenta de riscos, sobretudo por ser considerado como uma intervenção que consome bastante energia do paciente e, portanto, pode contribuir para a sua instabilidade clínica (TOLEDO LV, et al., 2019).

De modo geral, a equipe de enfermagem ao realizar o banho no leito de pacientes com "Déficit no autocuidado para banho" tende a executá-lo como uma atividade mecanizada, com base nos passos de um protocolo. Com isso, os riscos decorrentes de sua realização acabam sendo negligenciados (PRADO ARA, et al., 2017). Nesse contexto, emerge a necessidade de estimular e encorajar os profissionais da equipe de enfermagem a repensarem a sua assistência, sobretudo os seus padrões de referência para atividades como o banho no leito. Com isso, será possível uma assistência mais humanizada e eficaz, que ultrapasse os limites da execução de um cuidado marcado pela reprodução do saber teórico-prático (BACKES DS, et al., 2017)

Por fim, vale ressaltar a importância do banho como uma atividade onde a enfermagem pode, além de prestar cuidado a alguém que se encontra impossibilitado de cuidar de si, executar ações nos campos da prevenção e promoção da saúde do indivíduo (DIAS JAA, et al., 2016). 


\section{CONCLUSÃO}

Conclui-se que o diagnóstico de enfermagem "Déficit no autocuidado para banho" foi prevalente em todos os pacientes críticos avaliados, sendo caracterizado pela capacidade prejudicada de acessar a fonte de água; acessar o banheiro e pegar os artigos para banho. Entre os seus fatores relacionados, destacou-se a barreira ambiental. Tais achados confirmam as limitações dos pacientes críticos na manutenção da sua higiene corporal, sendo dependentes da equipe de enfermagem para a sua realização.

\section{REFERÊNCIAS}

1. BACKES DS, et al. Banheira portátil: tecnologia para o banho de leito em pacientes acamados. Rev Bras Enferm [Internet]. 2017; 70(2): 382-7.

2. BACKES MTS, ERDMANN AL, BUSCHER A. O ambiente vivo, dinâmico e complexo de cuidados em Unidade de Terapia Intensiva. Rev. Latino-Am. Enfermagem. 2015 maio-jun; 25(3): 411-8.

3. BENEDET SA, BUB MBC. Manual de diagnóstico de enfermagem: uma abordagem baseada na teoria das necessidades humanas básicas e na classificação diagnóstica da NANDA. Florianópolis: Bernúncia, 2. ed. rev. e ampl. 2001. 209p.

4. BEZERRA GKA. Unidade de Terapia Intensiva-Perfil das Admissões: Hospital Regional de Guarabira, Paraíba, Brasil. Revista Brasileira de Ciências da Saúde. 2013; 16(4): 491-496.

5. BISPO MM, et al. Risco de aspiração em pacientes críticos. Esc Anna Nery. 2016; 20(2): 357-362.

6. BRASIL. CONSELHO FEDERAL DE ENFERMAGEM - COFEN. Resolução COFEN n`358/2009, de 15 de outubro de 2009. Dispõe sobre a Sistematização da Assistência de Enfermagem e a implementação do Processo de Enfermagem em ambientes, públicos ou privados, em que ocorre o cuidado profissional de Enfermagem, e dá outras providências. Brasília, DF: COFEN; 2009.

7. CABRAL VH, et al. Prevalência de diagnósticos de enfermagem em unidade de terapia intensiva. Rev Rene. 2017; 18(1): 84-90.

8. CASTRO RR, et al. Perfil das internações em unidades de terapia intensiva adulto na cidade de Anápolis - Goiás 2012. Revista de Gestão em Sistemas de Saúde - RGSS. 2016; 5(2).

9. CHIANCA TCM, LIMA APS, SALGADO PO. Diagnósticos de enfermagem identificados em pacientes internados em Unidade de Terapia Intensiva Adulto. Rev Esc Enferm USP. 2012; 46(5): 1102-1108.

10. COSTA GS, et al. Banho no leito em cuidados críticos: uma revisão integrativa. Rev baiana enferm. $2018 ; 32$ : e20483.

11. DIAS JAA, et al. O banho no leito na óptica de estudantes de graduação em Enfermagem. J. res.: fundam. care. Online. 2016 out./dez; 8(4): 5087-509.

12. DIETRICH C, et al. Capacidade funcional em idosos e idosos mais velhos após alta da unidade de terapia intensiva. Coorte prospectiva. Rev Bras Ter Intensiva. 2017; 29(3): 293-302.

13. EULÁLIO MC, et al. Unidade de terapia intensiva: significados para pacientes em tratamento. Ciência\&Saúde. 2016; 9(3): 182-189.

14. FERREIRA AM, et al. Diagnósticos de enfermagem em terapia intensiva: mapeamento cruzado e Taxonomia da NANDA-I. Rev Bras Enferm [Internet]. 2016 mar-abr; 69(2): 307-15.

15. FONTELA PC, FORGIARINI JUNIOR LA, FRIEDMAN G. Clinical attitudes and perceived barriers to early mobilization of critically ill patients in adult intensive care units. Rev Bras Ter Intensiva. 2018; 30(2):187-194.

16. GUIMARÃES RM, et al. Diferenças regionais na transição da mortalidade por doenças cardiovasculares no Brasil, 1980 a 2012. Rev Panam Salud Publica. 2015; 37(2).

17. HERDMAN TH, KAMITSURU S. Nursing Diagnoses: Definitions \& Classification 2018-2020.Tenth Edition. Edited by; tradução: Regina Machado Garcez, Porto Alegre: Artmed, 2018. 1187p.

18. HORTA FG, et al. Ações de enfermagem prescritas para pacientes internados em um centro de terapia intensiva. Rev. Eletr. Enf. [Internet]. 2014 jul/set;16(3): 542-8.

19. LOPES JL, et al. Nursing guidance on bed baths to reduce anxiety. Rev Bras Enferm. 2015;68(3):437-43.

20. MAILLET JM, et al. Comparison of intensive-care-unit-acquired infections and their outcomes among patients over and under 80 years of age. J Hosp Infect. 2014; 87(3): 152-8.

21. MARTINS CP, et al. Diagnósticos de enfermagem em UTI: uma revisão integrativa. Rev. Aten. Saúde. 2018;16(57): 74-86.

22. MELO ACL, MENEGUETI MG, LAUS AM. Perfil de pacientes de terapia intensiva: subsídios para a equipe de enfermagem. Rev enferm UFPE on line. 2014 set; 8(9): 3142-8.

23. OKUMA SM, et al. Caracterização dos pacientes atendidos pela terapia ocupacional em uma unidade de terapia intensiva adulto. Rev. Interinst. Bras. Ter. Ocup. 2017; 1(5):574-588.

24. PEDRÃO TGG, et al. Diagnósticos e intervenções de enfermagem para pacientes cardiológicos em cuidados paliativos. Rev enferm UFPE on line.; 2018; 12(11): 3038-45.

25. PERÃO OF, et al. Características sociodemográficas e epidemiológicas de pacientes internados em uma unidade de terapia intensiva de adultos. Rev enferm UERJ. 2016; 25: e7736.

26. PORTO MLL, NÓBREGA MML, SANTOS SSC. Necessidades psicobiológicas e suas manifestações em idosos: revisão de literatura. RENE. 2005; 6(1): 125-134. 
27. POTTER PA. Fundamentos de Enfermagem. 9 ed. Rio de Janeiro: Elsevier, 2017. 893p.

28. PRADO ARA, et al. Banho no cliente dependente: aspectos teorizantes do cuidado de Enfermagem em reabilitação. Rev. Bras. Enferm. 2017;70(6):1337-42.

29. QUEIROZ F, REGO D, NOBRE G. Morbimortalidade na unidade de terapia intensiva de um hospital público. Revista Baiana de Enfermagem. 2013; 27(2): 164-171.

30. RAMOS GS, et al. Diagnósticos de enfermagem documentados em prontuários de pacientes em unidade de terapia intensiva. R. Enferm. Cent. O. Min. 2013; 3(2): 679-686.

31. RODRIGUEZ AH, et al. Características epidemiológicas e causas de óbitos em pacientes internados em terapia intensiva. Rev Bras Enferm [Internet]. 2016; 69(2): 229-34.

32. ROSIN J, et al. Identificação de diagnósticos e intervenções de enfermagem para pacientes neurológicos internados em hospital de ensino. Cienc Cuid Saúde. 2016; 15(4): 607-15.

33. SALOMÉ GM. Diagnóstico de enfermagem dos pacientes internados em uma Unidade de Terapia Intensiva. Saúde Coletiva. 2011; 8(47): 24-28.

34. SIQUEIRA ASE, SIQUEIRA-FILHO AG, LAND MGP. Análise do Impacto Econômico das Doenças Cardiovasculares nos Últimos Cinco Anos no Brasil. Arq Bras Cardiol. 2017; 109(1): 39-46.

35. SOUSA MAS, et al. Infecções hospitalares relacionadas a procedimentos invasivos em unidades de terapia intensiva: revisão integrativa. Rev Pre Infec e Saúde. 2017; 3(3): 49-58.

36. TANNURE MC, PINHEIRO AM. SAE: Sistematização da assistência de enfermagem: guia prático. 2 ed. Rio de Janeiro: Guanabara Koogan, 2011. 298p.

37. TOLEDO LV, et al. Efeitos oxi-hemodinâmicos de diferentes tipos de banho no leito em pacientes críticos: revisão sistemática. In: OLIVEIRA AC. O conhecimento na competência da teoria e da prática em Enfermagem. Atena editora. $2019 ; 3(1): 62-76$ 